\title{
Hidradenoma: A Skin Adnexal Tumour, Case Report and Literature Review
}

\author{
Kiran Kailas C, Nikhil Nanjappa* and Srikantaiah HC
}

Department of General Surgery, M.S. Ramaiah Medical College, Bangalore, India

*Corresponding author: Nanjappa N, Assistant Professor, Department of General Surgery, MS Ramaiah Medical College, Bangalore-560054, India, Tel: +91-9900280723; E-mail: drnikhilnanjappa@gmail.com

Received date: Nov 17, 2015; Accepted date: Dec 21, 2015; Published date: Dec 29, 2015

Copyright: (c) 2015 Nanjappa N, et al. This is an open-access article distributed under the terms of the Creative Commons Attribution License, which permits unrestricted use, distribution, and reproduction in any medium, provided the original author and source are credited

\begin{abstract}
Hidradenoma is a cutaneous, often benign tumour of the sweat glands. Also known by various other names such as nodular hidradenoma, eccrine acrospiroma, and solid cystic hidradenoma. A 41 year old man presented with a painless ulcero-proliferative polypoidal swelling over the right lower anterior abdominal wall. Histopathology of the wide local excision of the tumour revealed benign nodular hidradenoma. Benign Nodular Hidradenoma is known by many names such as solid cystic hidradenoma, clear cell myoepithelioma, eccrine sweat gland adenoma, large cell hidradenoma and eccrineacrospiroma. All patients are to be considered potential candidates for curative surgery. The management includes wide local excision with adequate margins to prevent recurrence. It must be differentiated from dermatofibrosarcoma protuberens as management and prognosis differs greatly.
\end{abstract}

\section{Introduction}

Hidradenoma is a cutaneous, often benign tumour of the sweat glands. Also known by various other names such as nodular hidradenoma, eccrine acrospiroma, and solid cystic hidradenoma. It is commonly seen in adults. It presents clinically as a solitary and firm intradermal nodule [1,2]. On Histopathological examination both solid and cystic components are present with characteristic clear cells, which contain a large amount of glycogen and thus appear clear under light microscopy.

Rapid growth and cutaneous ulceration clinically denote malignant transformation and histologically by an increased number of mitotic figures, nuclear atypia and angiolymphatic or perineural invasion. Atypical hidradenomas have frequent mitoses or nuclear atypia but lack clear evidence of asymmetric invasive growth. Such lesions require an aggressive management in view of a risk of recurrence and malignant transformation. In this case report, we describe a 41 year old male who presented with a swelling on the anterior abdominal wall, the excision biopsy of which turned out to be benign nodular hidradenoma.

\section{Case Report}

A 41 year old man presented with history of a painless swelling over the lower right anterior abdominal wall of 8 years duration. During which it had gradually progressed in size over the years but showed rapid progression in size in the past 6 months. No history of trauma. There was no pain associated with the swelling. No family history of any similar illness.

On examination a deeply pigmented pedunculated ulceroproliferative lesion was noted on the right lower anterior abdominal wall measuring $7.4 \mathrm{~cm}$ (Figure 1). The swelling was in the parietes and was freely mobile. The mass was non-pulsatile, non compressible, non reducible and no bruit was heard on auscultation. No other swellings were found elsewhere. A differential diagnosis of soft tissue sarcoma like dermatofibrosarcoma protuberans was considered so was hidradenoma.

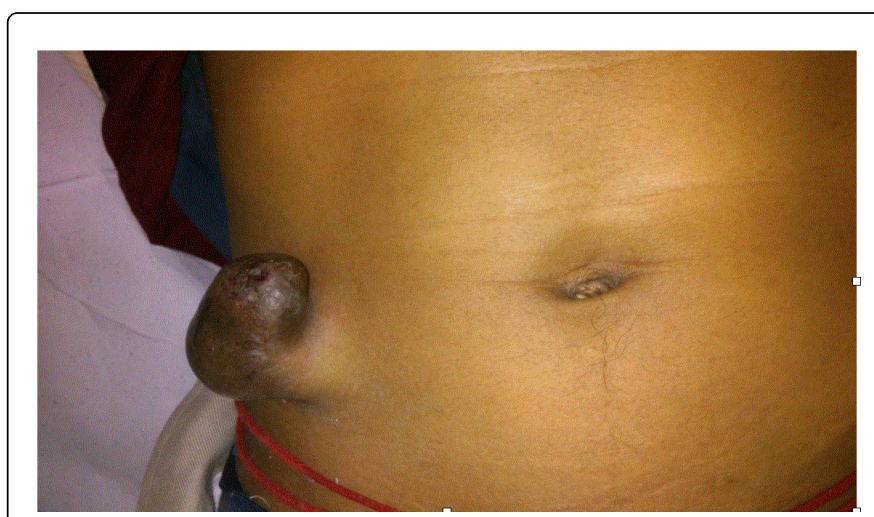

Figure 1: Ulcero-proliferative lesion on the anterior abdominal wall.

Fine needle aspiration cytology (FNAC) and core needle biopsy were done but were inconclusive. Ultrasonography (USG) abdomen and pelvis was done to evaluate the presence of any intra-abdominal pathology, but was unremarkable. Soft tissue scan of the anterior abdominal wall was done to evaluate the extension of the tumour into the abdominal wall and also to obtain the echogenic characteristics. It revealed a soft tissue tumour arising from the anterior abdominal wall with both solid and cystic components.

Wide local excision of the tumour was done with a $1 \mathrm{~cm}$ margin as malignancy was also possible and specimen was sent for histopathological examination (HPE). The incision site was closed primarily.

On HPE, the grossing showed skin covered soft tissue mass measuring $7.4 .3 \mathrm{~cm}$. The external surface shows a polypoidal growth measuring $4.3 .2 \mathrm{~cm}$. The cut surface showed solid and cystic areas filled with hemorrhagic fluid. Solid areas were grey white. 
The microscopy revealed that the resected skin margins were free from tumour. The sections showed a well circumscribed lobulated tumour predominantly in the dermis. The individual lobules showed two types of cells, i.e. basophilic and polygonal cells with clear cytoplasm. The nucleus was central, vesicular and had prominent nucleoli. Amidst these seen were tubular lamina lined by cuboidal cells. Occasional keratin pearl formation was noted. No evidence of atypical mitosis or necrosis seen. No epidermal connection was noted. The above features were consistent with benign nodular hidradenoma.

\section{Discussion}

Benign Nodular Hidradenoma is known by many names such as solid cystic hidradenoma, clear cell myoepithelioma, eccrine sweat gland adenoma, large cell hidradenoma and eccrineacrospiroma. It more often effects women. The common sites of occurrence are scalp, face, anterior trunk and extremities. There have been reported cases of Nodular Hidradenoma arising from various parts of the body, such as eyelids, vulva, palmar aspect of the hand, ulcerated lesion on the face and axillary tail of the breast. This tumour can occur at any age, but is commonly seen in the fourth decade of life. Skin changes such as changes in colour and thickness often accompany the lesion and may also be associated with serous or bloody discharge. It usually presents as a superficial dermal lesion and hence it is diagnosed early and often excised without much investigation.

Malignant transformation has been described and there is a report of metastasis in the skeleton from a lesion in the foot [3-5]. As they are dermal in origin, they seldom grow to enormous sizes. The differential diagnoses considered were hydatid cyst, soft tissue sarcoma, or desmoid tumour on the basis of clinical and radiographic examination. FNAC was attempted twice to establish a diagnosis before surgical removal but was inconclusive as it yielded only a hemorrhagic aspirate. True cut biopsy was attempted and was also inconclusive.

Excision of the swelling was quite straight forward as it was located on the anterior abdominal wall and was mobile. This tumour has been reported to rarely undergo malignant transformation [6]. However, there was no evidence of malignant transformation in this case as per the clinical, radiological and histopathological features. Malignant hidradenomas present with infiltrative growth pattern, deep extension, necrosis, nuclear pleomorphism, and $\geq 4$ mitoses per $10 \mathrm{HPFs}$. Malignant hidradenomas also exhibit Ki-67 $>11 \%$ and/or PHH3 $>0.7 \%$ [7]. The management of benign, atypical and malignant hidradenomas include wide local excision with adequate margins 0 (no consensus in literature as to the safe margin for wide local excision) to reduce the risk of recurrence.

Clinically, the swelling appears to be dermatofibrosarcoma protuberens. Due to its mode of presentation and appearance, a $1 \mathrm{~cm}$ margin was given while excising the lesion to account for that. dermatofibrosarcoma protuberens is a malignant tumour that has poorer prognosis. Hence all efforts must be made to obtain preoperative histopathological diagnosis. However if it is not possible due to any reason, one must err on the side of safety and perform a wide local excision with appropriate margins. This would especially hold good for in situations where clearance would not be difficult. A large dermatofibrosarcoma protuberens might even require post-operative radiotherapy. So differentiation is imperative.

\section{References}

1. Elder D, Elentisas R, Ragsdale BD (1997) Tumors of the epidermal appendages, in David E (ed): Lever's Histopathology of the Skin, Philadelphia: LippincottRaven Publisheres, 47804.

2. Wilhelmi BJ, Appelt EA, Phillips LG (1999) A rare case of atypical eccrine acrospiroma of the scalp and a literature review. Ann Plast Surg 42: 568-569.

3. Biddlestone LR, Mclaren KM, Tidman MJ (1991) Malignant hidradenoma: a case report demonstrating insidious histological and clinical progression. Clin Exp Dermatol 16: 474-477.

4. Dumont K, Humph rays T, Malhotra R: (1996) Stump the experts. Dermatol Rev. 22: 998-999.

5. Ogilvie JW (1982) Malignant eccrine acrospiroma. A case report. J Bone Joint Surg Am 64: 780-782.

6. Tony B, Stephen B, Neil C, Christopher G. (2010) Rook's Textbook of Dermatology Wiley-Blackwell. (8th ed) 21: 37-22

7. Nazarian RM, Kapur P, Rakheja D, Piris A, Duncan LM, et al. (2009) Atypical and malignant hidradenomas: a histological and immunohistochemical study. Mod Pathol 22: 600-610. 